\title{
Modelling of handover of multimedia traffic in mobile satellite communication
}

\begin{abstract}
This paper presents the handover management in mobile satellite communications with multimedia traffic. Multimedia traffic is becoming the next hop in mobile satellite communication, due to its demand. The coverage of the satellite network knows no boundary and with the concept of the broadband satellite network, communication for multimedia and high-data rate multimedia communications can be provided. The handover initiation and execution is proposed with a queuing algorithm in the on-board processing (OBP) technique. The traffic is generated according to its nature of burstiness to represent the traffic behaviour on variation of the channels. The modelling could benefit network operators to fully conserve the frequency spectrum and to increase capacity.
\end{abstract}

Keyword: Mobile satellite communications; Multimedia traffic 